\title{
Comparison of three different methods for the detection of circulating tumor cells in mice with lung metastasis
}

\author{
WEIFENG XU ${ }^{1,2}$, BING WU $^{1-3}$, LENGXI FU $^{1-3}$, JUNYING CHEN $^{1-3}$, ZENG WANG $^{1-3}$, FEI HUANG $^{1-3}$, \\ JINRONG CHEN ${ }^{1-3}$, MEI ZHANG ${ }^{4}$, ZHENHUAN ZHANG $^{4}$, JINGAN LIN $^{1-3}$, RUILONG LAN $^{1-3}$,

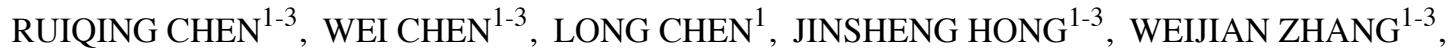 \\ YUXIONG DING ${ }^{1-3}$, PAUL OKUNIEFF ${ }^{4}$, JIANHUA LIN ${ }^{1-3}$ and LURONG ZHANG ${ }^{1-4}$
}

${ }^{1}$ First Affiliated Hospital of Fujian Medical University; ${ }^{2}$ Fujian Key Laboratory of Individualized Active Immunotherapy;

${ }^{3}$ Key Laboratory of Radiation Biology of Fujian Province Universities, Fuzhou, Fujian 350005, P.R. China;

${ }^{4}$ Department of Radiation Oncology, University of Florida, Gainesville, FL 32610, USA

Received October 27, 2016; Accepted April 5, 2017

DOI: $10.3892 /$ or.2017.5613

\begin{abstract}
Circulating tumor cells (CTCs) represent the key step of cancer cell dissemination. The alteration of CTCs correlates with the treatment outcome and prognosis. To enrich and identify CTCs from billions of blood cells renders a very challenging task, which triggers development of several methods, including lysis of RBC plus negative or positive enrichment using antibodies, and filter membrane or spiral microfluidics to capture CTCs. To compare the advantages of different enrichment methods for CTCs, we utilized the 4T1 breast cancer cells transfected with both green fluorescent protein (GFP) and luciferase to trace CTCs in the experimental lung metastasis model. Three methods were used to detect CTCs at the same time: bioluminescence assay, smearing method, and membrane filter method. The in vivo alive mouse imaging was used to dynamically monitor the growth of lung metastases. The sensitivity and accuracy of three detection methods were compared side-by-side. Our results showed that 1) the sensitivity of bioluminescence assay was the highest, but there was no information of CTC morphology; 2) the smearing method and membrane filter method could observe the detail of CTC morphology, such as in single or in cluster, while their sensitivity was lower than bioluminescence assay; 3) A dynamic observation at a 7-day intervals, the lung metastatic
\end{abstract}

Correspondence to: Dr Lurong Zhang or Dr Jianhua Lin, First Affiliated Hospital of Fujian Medical University, Fuzhou, Fujian 350005, P.R. China

E-mail: 1z8506@163.com

E-mail: jianhual@126.com

Abbreviations: CTCs, circulating tumor cells; GFP, green fluorescence protein; RBC, red blood cells; WBC, white blood cells; DAPI, 4',6-diamidino-2-phenylindole dihydrochloride; i.v. injection, intravascular injection

Key words: circulating tumor cells, comparison of three detection methods, lung metastases cancer grew at a log speed, while CTCs were increased at a low speed. This might be due to the activated immune cells eliminating the CTCs at a speed much faster than CTCs were generated. This comparison of three CTC detection methods in mouse model suggests that bioluminescence assay could be used in quantitative study of the effect of certain agent on the suppression of CTCs, while GFP-based morphological assays could be used to study the dissemination mechanism of CTCs. The combination of both bioluminescence assay and GFP-based assay would generate more information for quantity and quality of CTCs.

\section{Introduction}

Metastasis is the major cause of cancer death (1). Although the cancer dissemination is a complicated process, involving detachment of cancer cells from primary site, intravasation, extravasation and seeded in a remote site, the existing of circulating tumor cells (CTCs) is one of land-marks for metastasis process (2-7). While the 'seed-soil' concept is well-accepted for a successful metastasis, it is not doubted that the numbers of CTCs reflect several aspects related with the cancer malignancy and treatment outcome $(8,9)$. A high numbers of CTCs are likely to indicate a high potential of metastasis and a possible failure of cancer treatment with poor prognosis (10). The continuous drop in the CTC number might suggest a correct regimen of anti-cancer treatment and a good outcome for patients. Thus, the determination of CTCs has attracted great attention in past two decades and is called 'liquid biopsy' (11).

However, the major obstacle for utilization of this index raises from the nature of CTCs, which is a very rare event in whole blood tested, especially in the early stage of cancer, the best treatment window for cancer. Technically, to capture and detect a single digit of CTC from $10^{9} / \mathrm{ml}$ blood cells is very challenging. Few assays pass FDA regulation and are used in clinical practice.

The principles of defining a few of CTCs from huge numbers of normal blood cells are based on: 1) physical properties of CTCs: cell diameter, density and charge; 
2) biological characteristics of CTCs: the unique epithelial marker of CTCs (such as cytokeratin and EpCAM), tumor specific proteins and antigens, chromosome heteroploid or RNA abnormality. Based on these physical and biological characteristics of CTCs, several methods have been developed to accomplish two major steps of determination of CTCs: enrichment and detection.

For the first step of enrichment, several methods have been used with their advantages and disadvantages: 1) Antibodybased method: the best example is the FDA approved CellSearch method, which uses anti-EpCAM to capture/immobilize the CTCs as positive selection (12-14). CTCs chips use the same principle (15). Other immune magnetic separations utilize anti-CD45 to capture and then discard the WBC after lysis of $\mathrm{RBC}$ as negative selection (13). The problem encountered by positive selection is that the cancer cells might have a transition from epithelium to mesenchymal cells (EMT) and loss of the epithelial marker, resulting in the low detection rate and false negative. For the negative selection, the CD45 might not stain well for all non-tumor cells in blood, such as macronuclei cells (precursor of platelet) or detached endothelium or transformed WBC, resulting in a false positive. 2) Physics-based method: the membrane filter method utilizes the fact that the sizes of CTCs are bigger than most of normal blood cells $(16,17)$; the centrifugation and /or microfluidic separation methods utilize the unique density, size and/or charge of CTCs. These methods require sophisticated techniques and special devices.

For the second step of detection, several methods are also utilized: 1) nucleotide-based assay: FISH assay is able to reveal the chromosome heteroploid or RNA abnormality, and the RT-PCR reflects the overexpression of oncogenes (18); 2) Antibody-based assay: the use of anti-CK can detect the epithelial origin CTCs by flow cytometry (19) or immunocytochemistry staining. The key issues in the detection step are the specificity of reagents, the sample treatment to expose the target and the interferences in the amplification process. Although the different techniques have been tested and used, their specificity and sensitivity are not ideal (20-22), and the side-by-side comparison of their characteristics is needed.

Animal model has been utilized to study tumor metastasis for a long time, due to 1) the heterogeneity is slight in a small animal model; 2) the conditions of different types of animal experiments are easy to control. Similarly, animal models can be utilized to study the alteration patterns of CTCs varied with the stage development of metastasis and the different treatments. However, how to detect mouse CTC alterations and which is the best method to accurately reflect the CTC alterations are under studied, since the most attention is paid to detection of human CTCs, but not in animal models.

In this study, we utilized the dual-gene transfected $4 \mathrm{~T} 1$ breast cancer cell line, which stably expressed the GFP and luciferase for accurately tracking and quantitating CTCs, to perform a side-by-side comparison of three different CTC assays for their advantages and disadvantages. The same amounts of blood sample from BABL/c mice with lung metastases as result of intravenous injection of GFP and luciferase dual-labeled $4 \mathrm{~T} 1$ cells were subjected to three different procedures, i.e. after lysis of RBC, 4T1-CTCs were detected in three formats: 1) smeared blood samples after lysis of RBC on slides and counted for GFP-4T1; 2) quantitated luciferase-4T1 with bioluminescence assay; 3) captured CTCs from blood on filter membrane and counted for GFP-4T1. The goal of this study was to determine: 1) Which method is more sensitive in reflecting CTCs; 2) What is unique in each method; and 3) Whether the filter method lose more CTCs. This study will help us to decide which method should be chosen for different research purposes.

\section{Materials and methods}

Reagents and instruments. Fetal bovine serum (FBS) was purchased from Zhejiang Tianhang Biotechnology Co; Ltd. D-Luciferin was purchased from BioVision (Milpitas, CA, USA); DAPI, 4',6-diamidino-2-phenylindole dihydrochloride (cat\# D8417, Sigma Aldrich Co., USA); Whatman ${ }^{\mathrm{TM}}$ Nuclepore $^{\mathrm{TM}}$ Polycarbonate Track-Etched Membranes with $8 \mu \mathrm{m}$ pore size was purchased from Whatman (cat\# 09-300-57, Pittsburgh, PA, USA); Bioluminescence signal of lung metastases was quantitatively measured by IVIS Spectrum (PerkinElmer Co., Waltham, MA, USA). Firefly Luciferase Reporter gene assay kit was purchased from Beyotime Biotechnology Co., Ltd. Relative light unit (RLU) was measured by luminometer (Antu, Zhengzhou, China). BX63 fluorescence microscope was from Olympus, Center Valley, PA, USA.

Cell lines and cell culture. GFP-Luc-4T1 dual labeled cell line was purchased from Caliper Life Science (Hopkinton, MA, USA) and cultured in RPMI-1640 medium containing $10 \%$ FBS, $100 \mathrm{U} / \mathrm{ml}$ of penicillin and streptomycin.

Experimental animals. Female BALB/c mice were purchased from Slaccas Experimental Animal LLC (license\# SCXK 2012-0002, Shanghai, China). Animal studies were approved by Fujian Medical University Institutional Animal Care and Use Committee.

Establishment and evaluation of an experimental mouse model for breast cancer lung metastasis. GFP-Luc-4T1 cells $\left(10^{6}\right)$ were freshly collected, suspended in $300 \mu 1$ PBS, and then injected into the tail vein of BALB/c mice. PBS was injected into the tail vein of another nine BALB/c mice as control group. The growth of pulmonary metastasis was observed by IVIS Spectrum on day 7, 14, 21, and the data were analyzed by Spectrum Living Image 4.0 software. Lung tissue with metastases of GFP-Luc-4T1 were removed and stained by haematoxylin and eosin (H\&E) after the mice were sacrificed on day 21.

Collection of blood samples for CTCs. As shown in Fig. 1A, on day 7, 14, 21 after GFP-Luc-4T1 i.v. injection, $300 \mu 1$ whole blood samples were collected by tail vein bleeding into K3-EDTA-coated tubes, mixed well and then equally divided into three $2 \mathrm{ml}$ tubes, marked A, B, C, respectively, for assessment of CTCs with three different methods.

CTCs detected with luciferase assay. As shown in Fig. 2A, $100-\mu 1$ blood was centrifuged at $92 \times \mathrm{g}$ for $5 \mathrm{~min}$, and plasma was discarded. Cell lysis buffer $(200 \mu \mathrm{l})$ (Beyotime Biotechnology Co., Ltd.) was added to resuspend cells, 

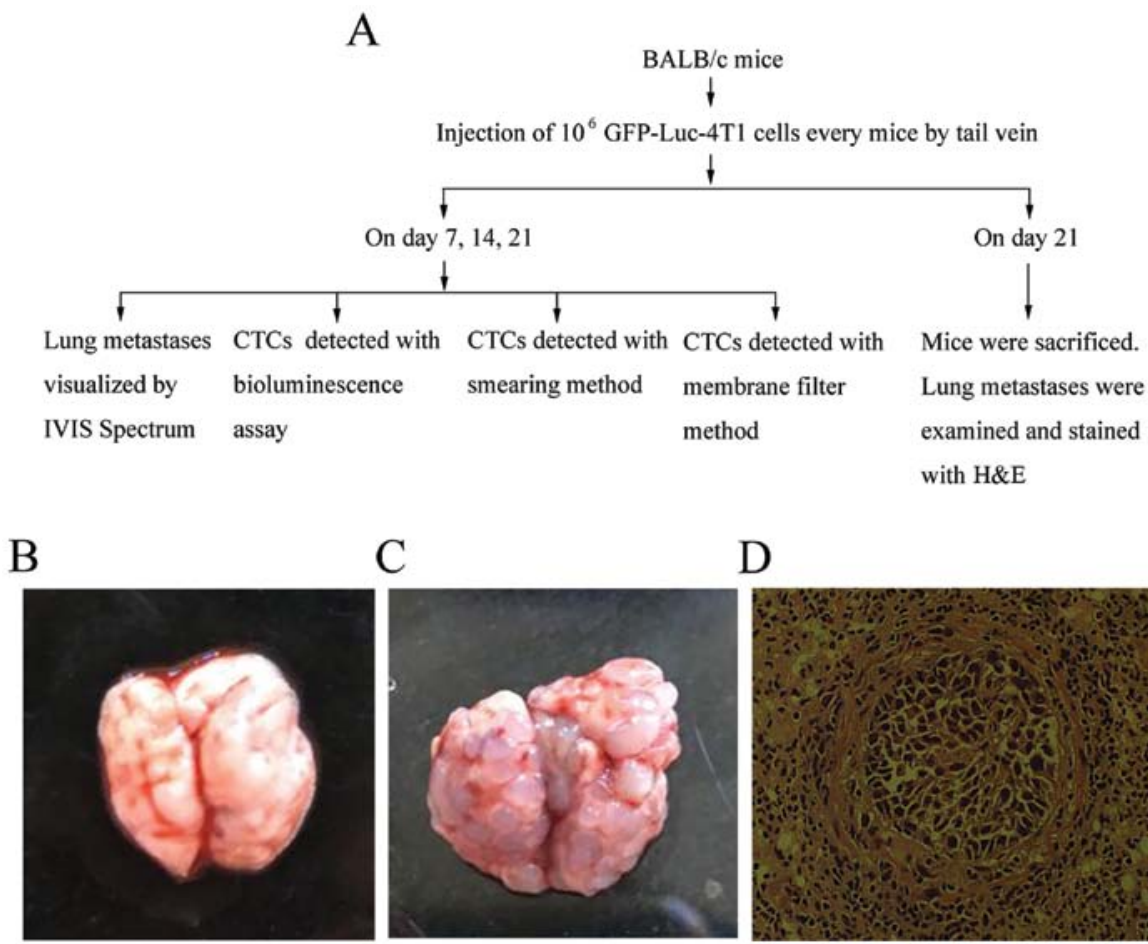

\section{D}

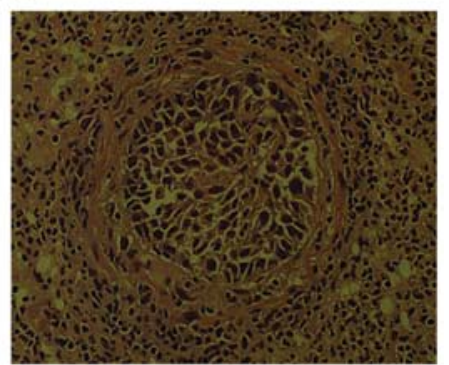

Figure 1. Schematic of experimental design and establishment of GFP-Luc-4T1 experimental metastasis model in BALB/c mouse. (A) Schematic of experimental procedures; (B) No lung metastases in PBS control mice on 21 days after inoculation. (C) Full of lung metastases in GFP-Luc-4T1 cell-bearing mice on 21 days after inoculation. (D) Lung metastases were stained by haematoxylin and eosin (H\&E) (x100).

A

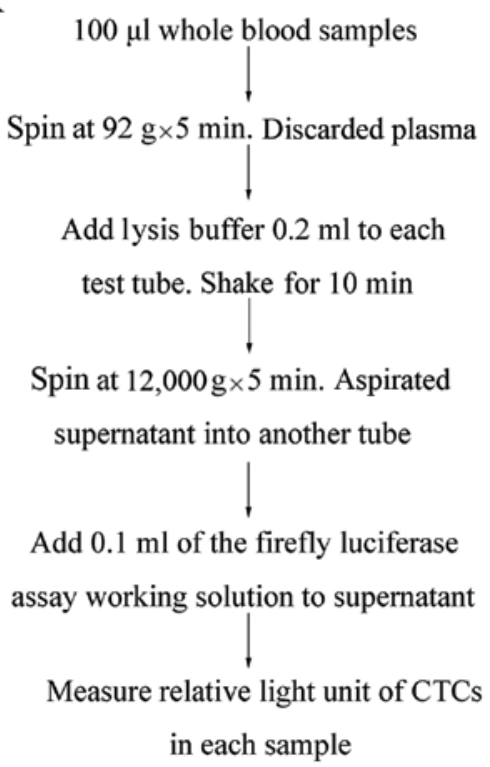

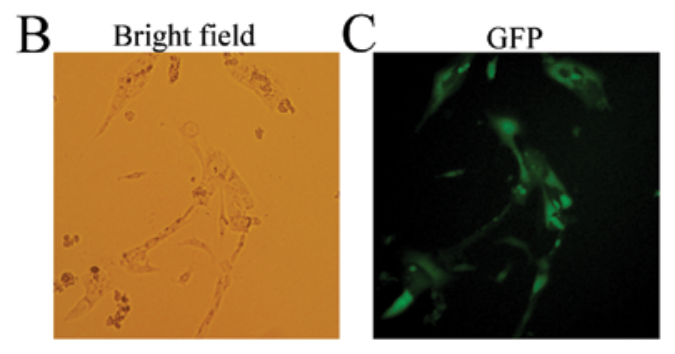

$\mathrm{D}$

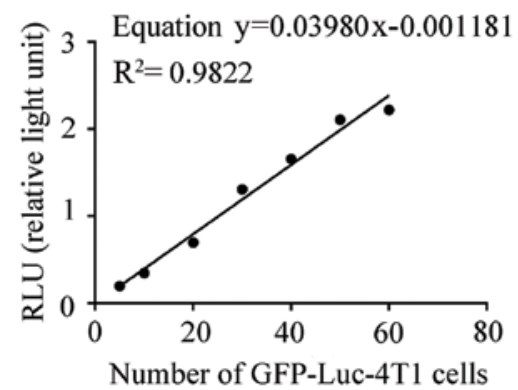

Figure 2. CTC detection by bioluminescence assay. (A) Schematic of experiment procedures. (B) Images of GFP-Luc-4T1 cells under the bright field (x200). (C) Images of GFP-Luc-4T1 cells under the $488 \mathrm{~nm}$ excitation (x200). (D) The standard curve, reflecting the corresponding relationship between the number of GFP-Luc-4T1 cells and the RLU value.

incubated at room temperature for 8-10 min with occasional shaking, then centrifuged at $12,000 \mathrm{x} g$ for $5 \mathrm{~min}$. The supernatant was aspirated into another tube and $100 \mu \mathrm{l}$ of luciferin working solution (Beyotime Biotechnology Co., Ltd.) was added to the supernatant, reacting with luciferase of CTCs of GFP-Luc-4T1. Immediately, the RLU in each tube was assayed by luminometer (Antu LLC, Henan, China).
To set up standard curve, the freshly harvested GFP-Luc-4T1 cells were counted, then 0, 5, 10, 20, 30, 40, 50, 60 cells were added to tubes, respectively. RLU was assayed according to the above method and the derived equation was used as standard curve to calculate the CTC numbers of GFP-Luc-4T1 cells from mice with experimental lung metastases. 
A

$100 \mu \mathrm{l}$ whole blood samples

lysis buffer. Shake for $10 \mathrm{~min}$

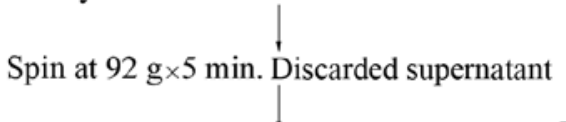

Stain with DAPI for $15 \mathrm{~min}$

Smear cells 'on glass slide

Cover with cover glass

Measure CTCs under

fluorescent microscope
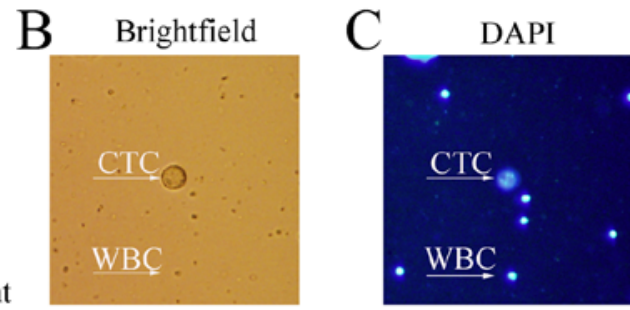

D

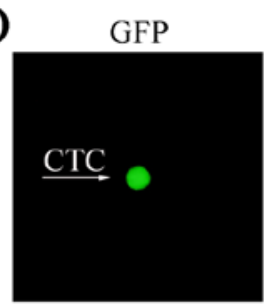

$\mathrm{E}$

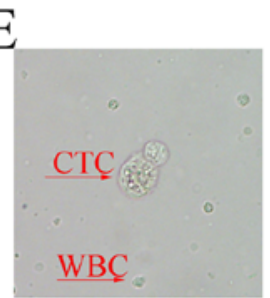

F

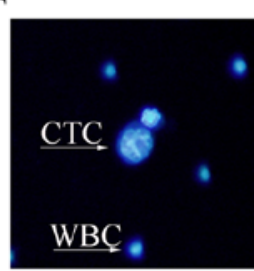

G

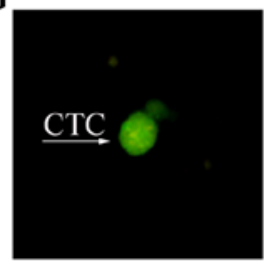

Figure 3. CTC detection by smearing method. (A) Schematic of the experiment procedures. Observation CTCs by smearing method on day 14 (x200) under bright field (B), under excitation light with DAPI stain (C), under excitation light with GFP stain (D). Observation CTCs by smearing method on day 21 (x400) under bright field (E), under excitation light with DAPI stain (F), under excitation light with GFP stain (G).

A

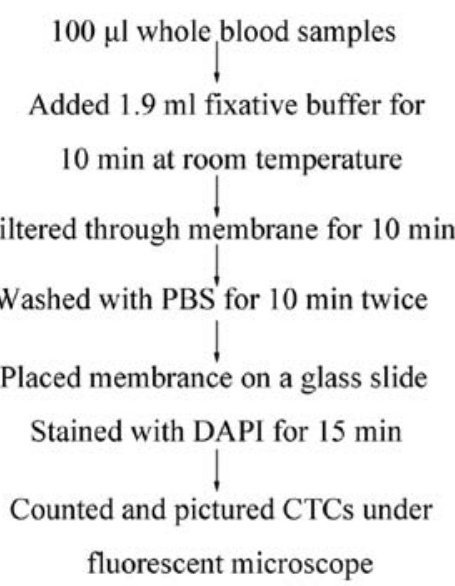

$\mathrm{B}$

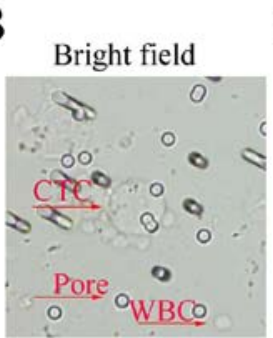

$\mathrm{E}$

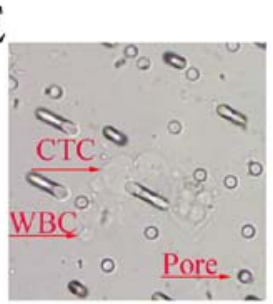

C
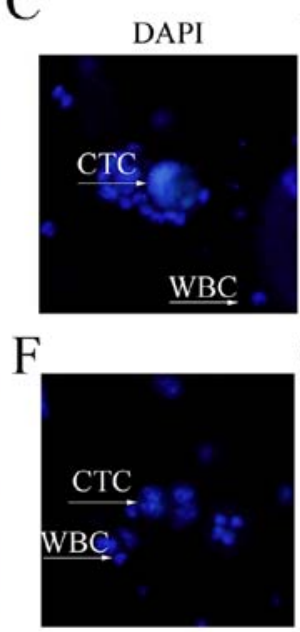

$\mathrm{D}$

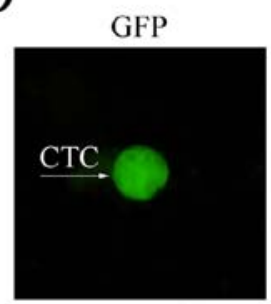

G

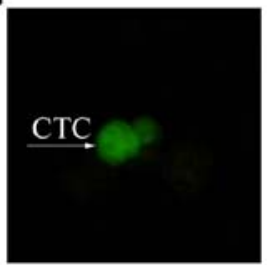

Figure 4. CTC detection by membrane filtration. (A) Schematic of experiment procedures. Observation CTCs by membrane filtration on day 14 (x400) under bright field (B), under excitation light with DAPI stain (C), under excitation light with GFP stain (D). Observation CTCs by membrane filtration on day 21 (x400) under bright field (E), under excitation light with DAPI stain (F), under excitation light with GFP stain (G)

CTCs detected with counting on the slide. As shown in Fig. 3A, $1.9 \mathrm{ml}$ of RBC lysis buffer was added to $100 \mu \mathrm{l}$ blood, incubated at room temperature for 8-10 min with occasional shaking, then centrifuged at $92 \times \mathrm{g}$ for $5 \mathrm{~min}$. After aspiration of supernatant without disturbing pellet, $200 \mu 1$ of $3 \mathrm{nM}$ DAPI in PBS was added to the tube well resuspend cell pellet, smeared on $24 \times 50 \mathrm{~mm}^{2}$ glass slide 15 min later, covered with $0.13-0.17$ thick cover glass. The GFP/DAPI positive 4T1 CTCs were pictured and counted under BX63 Olympus fluorescence microscope. The $3 \mu \mathrm{M}$ DAPI stock solution was made in the staining buffer of $100 \mathrm{mM}$ Tris, $\mathrm{pH} 7.4,150 \mathrm{mM} \mathrm{NaCl}, 1 \mathrm{mM} \mathrm{CaCl} \mathrm{Cl}_{2}, 0.5 \mathrm{mM}$ $\mathrm{MgCl}_{2}, 0.1 \%$ Nonidet P-40.

CTCs detected with counting on filter membrane. As shown in Fig. 4A, $1.9 \mathrm{ml}$ of fixative buffer $(0.2 \%$ paraformaldehyde, $0.0372 \%$ EDTA, and $0.1 \%$ bovine serum albumin) was added to $100 \mu \mathrm{l}$ blood for $10 \mathrm{~min}$ at room temperature. Then, the $2 \mathrm{ml}$ fixative buffer was placed in a 5-ml syringe connected to a filter supporter with $8 \mu \mathrm{m}$ pore size of Polycarbonate Track-Etched Membrane, and passed the filter membrane in $10 \mathrm{~min}$. After washing with $2 \mathrm{ml}$ of PBS twice at the same speed, the membrane was placed on a glass slide as support. Of $3 \mathrm{nM}$ DAPI in PBS, $200 \mu \mathrm{l}$ was added to the membrane, then covered with cover glass. The numbers of GFP/DAPI positive 4T1 CTCs were counted, and images were pictured by BX63 Olympus fluorescent microscope.

Statistical analysis. GraphPad Prism6 software was used for statistical analysis, and measurement data are expressed as mean \pm standard deviation. An unpaired, t-test was used for comparing two groups of measurement data, and the difference was statistically significant, at $\mathrm{P}<0.05$. 
A

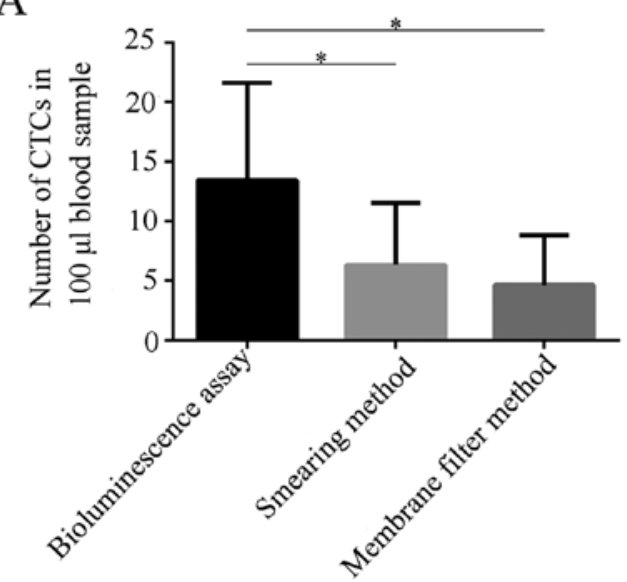

B

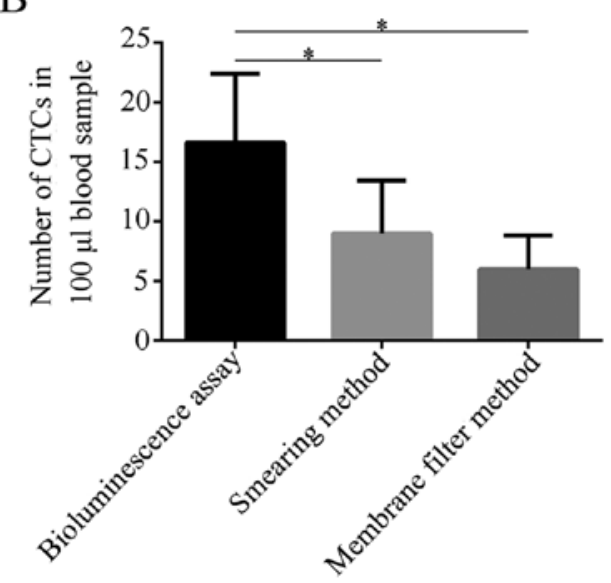

Figure 5. Comparison of CTC detection by three methods. (A) Comparison of CTC detection by three methods on day 14. (B) Comparison of CTC detection by three methods on day 21 . $^{*} \mathrm{P}<0.05$.

\section{Results}

Establishment and evaluation of pulmonary metastasis of $4 T 1$ breast cancer in $B A L B / c$ mouse model. In this study, the 1st key step was to set up the experimental lung metastasis model. After injection of $1 \times 10^{6}$ mouse GFP-Luc-4T1 cells into BALB/c syngeneic mice, the breast cancer cells were able to settle down in lung and grew with time. In addition, the growth of 4T1 lung metastases was evidenced by seeing the tumor nodules increase with time. While none of lung metastases could be seen in the control PBS group (Fig. 1B), the tumor nodules were full in the lung of the GFP-Luc-4T1 cells injected mice on day 21 (Fig. 1C), which could cause death due to respiratory failure of lung cancer. Finally, at the end of study, the 4T1 metastases could be seen fully occupied in lung tissues (Fig. 1D). All evidence supported that this experimental lung metastasis model was successful, which could provide continuous CTCs for the assessment with three different CTC assays.

Luc-CTCs quantitatively detected with luciferase assay. The GFP-Luc-4T1 cells used in this study are stable and highly expressed in both GFP and luciferase. All living cells that could be seen under a bright field of regular inverted microscope (Fig. 2B) were GFP positive under an inverted fluorescent microscope (Fig. 2C). Since both GFP and luciferase are driven by a same promoter, the luciferase is also likely to be expressed by each of 4T1 cells. The RLU efficiency due to the catalysis of luciferin by luciferase produced by $4 \mathrm{~T} 1$ cells was proportional to the cell number, which could well serve as standard for counting CTCs (Fig. 2D). The equation was $\mathrm{Y}=0.03980 \mathrm{x}-0.001181$ and the correlation coefficient $R^{2}$ was 0.9822 . Two trace markers carried by GFP-Luc-4T1 cells are unique with no background in BALB/c mice, which provides a clear identity for us to detect and count the CTCs in a precise fashion.

As shown in Fig. 2A, we could lyse all the cells in $100 \mu \mathrm{l}$ of blood, then added D-luciferin to the lysed CTCs for their luciferase activity. From the numbers of photo RLU, we were able to dynamically monitor the alteration of CTCs with time in the mouse model. Due to the enzymatic reaction having amplification effect, luciferase assay could be the most sensitive assay in reflecting the existing CTCs as compared to other two assays with counting GFP positive CTCs one by one with nude eye under fluorescent microscope, which was confirmed by Figs. 5 and 6E.

GFP-CTCs visualized on a slide. As shown in Fig. 3A, after lysis of RBCs, the CTCs were counter-stained with DAPI/ anti-fader to exclude the no-cell green fluorescence debrides, then the re-suspended cell pellet was smeared on the slide, and covered with 50\% glycerol-PBS for observation under a fluorescent microscope. This simple process would also allow us to follow-up the CTC alterations with times. The disadvantage is the high background of 'ghost cell' (membranes of lysed RBCs) debris as shown in Fig. 3C compared to the filter membrane method (Fig. 4C). We found that during the first 14 days, the CTCs were seen mostly in a single cell (Fig. 3B-D), however, after 14 days, we could see two cancer cells together as clusters (Fig. 3E-G), which might have a special property in the cancer progression.

CTCs assessed on filter membrane. When the fixed CTCs containing blood cells in the synergies were pushed through $8 \mu \mathrm{m}$ pore size of Polycarbonate Track-Etched Membrane at a speed of $2 \mathrm{ml} / 10 \mathrm{~min}$, the small RBCs and most of WBCs passed the pores, while the CTCs were retained on the top of membrane due to their big size. Similar to that seen in smeared slides, in week 2, CTCs existed mostly as single cells (Fig. 4B-D), while in week 3, some CTCs were seen in clusters (Fig. 4E-G).

Comparison of three methods. The sensitivity of CTCs detected by three methods described above was compared at 14 and 21 days after inoculation. As shown in Fig. 5A, on day 14 , the CTCs in same amount of blood detected by bioluminescence assay had the highest number as compared to the smear method $(\mathrm{P}=0.0426)$ and membrane filtration method $(\mathrm{P}=0.0110)$, while there was not statistical difference between smear method and membrane filtration $(\mathrm{P}=0.4632)$. A similar pattern was observed on day 21 (Fig. 5B). This set of data indicates that the bioluminescence assay has a high sensitivity in detecting CTCs, which might relate with the enzymatic amplification of luciferase. 


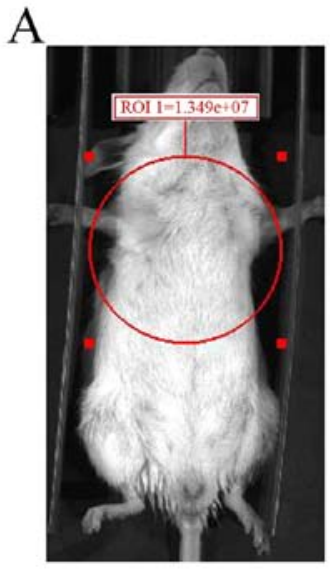

Day 7

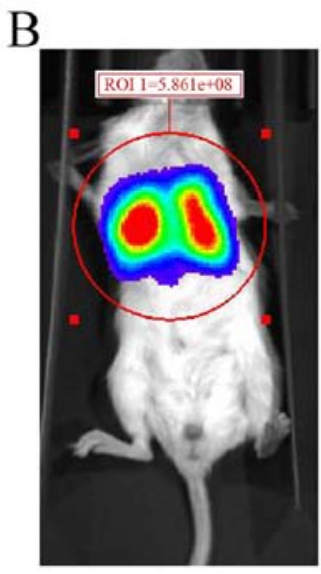

Day 14

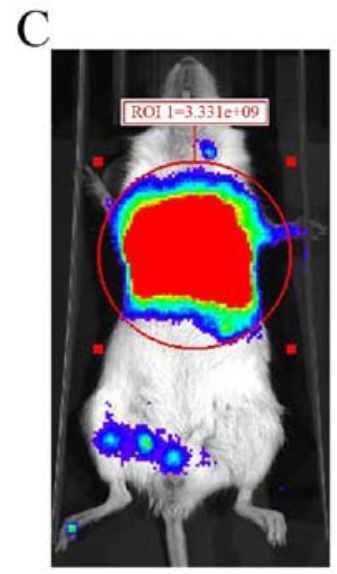

Day 21

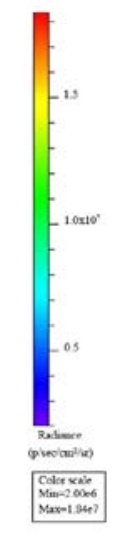

$\mathrm{E}$

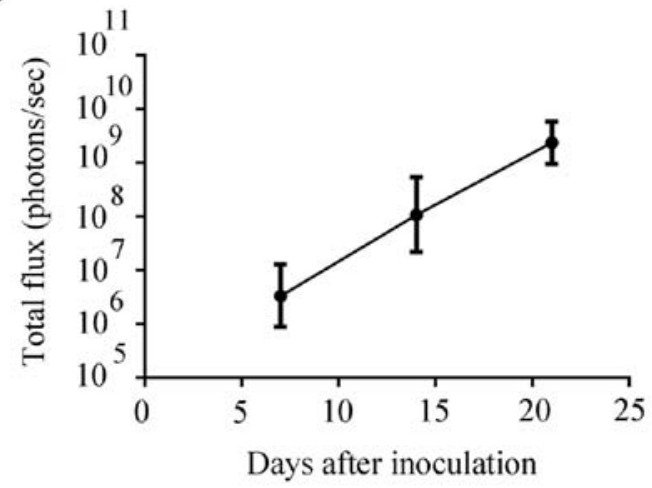

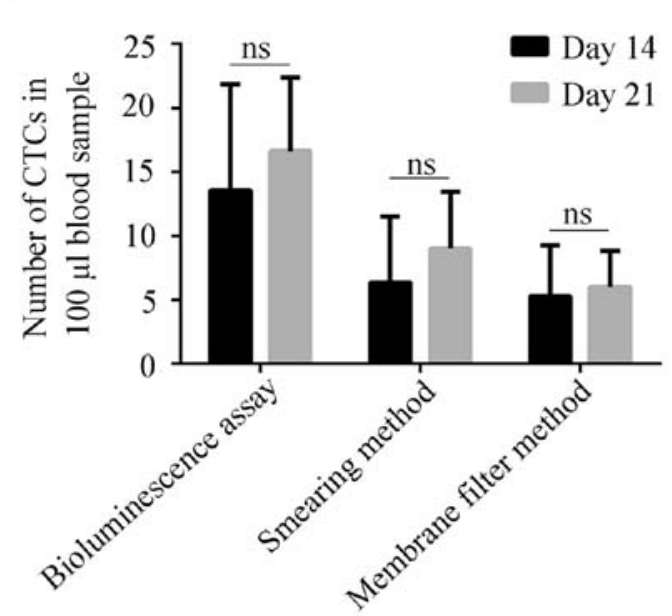

Figure 6. The correlation between the dynamic changes of the number of CTCs and the growth of lung metastases. The growth of lung metastases as monitored using bioluminescence imaging on day 7 (A), 14 (B), 21 (C). (D) Corresponding quantification of bioluminescence imaging signal on day 7, 14, 21. (E) Comparison of CTC detection by three methods at two time points (day 14, 21). $\mathrm{P}<0.05$; ns, not significant.

The increase of tumor cells in lung metastatic site was faster than CTCs. To our surprise, when comparing the speed of increase in lung metastases with that of CTCs with time, the in vivo bioluminescence imaging signal (measured by IVIS Spectrum) representing size of lung metastases increased at the log speed (bioluminescence imaging signal on day 7 , $7.507 \times 10^{6} \pm 3.885 \times 10^{6}$; on day $14,2.615 \times 10^{8} \pm 1.001 \times 10^{8}$; on day $21,3.198 \times 10^{9} \pm 7.959 \times 10^{8} ; \mathrm{P}<0.001$, Fig. $6 \mathrm{D}$ ), while the increase of speed of CTCs was only a few cells in 7 day intervals even detected by the sensitive bioluminescence assay $(\mathrm{P}=0.3748$, Fig. $6 \mathrm{E})$. We speculate that this might relate to a balance between the number of cancer cells entering the circulation and their clean-up by the immune cells.

\section{Discussion}

This study focused on defining good assays for detecting CTCs in a tumor-bearing mouse model (23), which might be of benefit: 1) to reveal the process of metastasis; 2) to study how the host disseminated CTCs; and 3) to search for effective agents against tumor metastasis.

The CTC assay for experimental mouse model is different from that for human, since the mouse cancer cells could be labeled with biomarkers, such as fluorescence protein and/or luciferase, while human cancer cells exist in an unlabeled native form. Using the labeled mouse CTCs, we can determine advantages and disadvantages of the three different assays for their efficacy in quantification of CTCs.

Utilizing the unique dual labeled GFP-Luc-4T1 cells, we were able to compare three assays that practically could be used in monitoring the CTCs in a mouse model. Our new findings are: First, bioluminescence assay is the most sensitive assay for CTC detection as compared to other two methods by counting CTCs one by one with nude eye (Figs. 5 and 6E). This is mainly due to the luciferase catalytic effect on luciferin, an enzymatic amplification of signal that can be sensitively measured with a luminometer. The advantages of this assay are: 1) it is capable of dealing with relative large volume of blood, increasing the chance of capturing more CTCs; 2) it requires simple procedures, only lysis of RBCs and addition of luciferin to cell pellet; 3 ) its reading result with luminometer is subjective without human error; 4) it is very specific and a sensitive assay. Sasportas et al reported that bioluminescence assay could detect 2 CTCs in $100 \mu$ l blood (24). Some studies show that bioluminescence imaging is 3-8-fold more sensitive than fluorescence imaging $(25) ; 5)$ it has very low background, 
since there is no luciferase background in living mammals, which yields a high signal/noise ratio, enhancing its sensitivity. However, the limitation of this method is that the number of CTCs is calculated from standard curve, therefore, there are some errors and the accuracy is not high. In addition, this method could not reflect morphological characteristics of CTCs since all CTCs are lysed as whole.

Secondly, although GFP-based two assays (CTCs smeared on slide or captured with filter) are less sensitive compared to the bioluminescence assay and consume much more human labor, they allow us to trace GFP marker of CTCs and observe the morphological characteristics of CTCs. Before day 14, the CTCs existed mainly as a single cell, however, after 14 days approximately $10 \%$ of CTCs were two together as CTC clusters, consistent with the report of Choi et al (26). This information is particularly important, since it is believed that CTC-clusters have 23-50-fold increased metastatic ability (27). Besides, detection of the CTC-clusters, they have some more advantages: 1) they are simple and convenient assays, easy to train the operators; 2) they do not need other expensive instruments, so that they can be used in general laboratory. However, the two assays have some disadvantages: 1) it is time- and manpower-consuming with human counting errors; 2) during the long-term growth process of tumor cells, a part of tumor cells could lose their GFP, which means that the green fluorescence of some CTCs are weaken with time, which might cause false negative; 3 ) some unrelated impurities also can emit green fluorescence, which might generate false positive. To overcome this, we used DAPI staining in the cell nucleus to eliminate interferences of non-cell impurities. It should be pointed out that when using 'smeared CTCs on slide assay', the cell drop should be spread thin enough to avoid crumbles of ghost cells covering the CTCs. When using the filtering method, the force of pushing blood cells passing the filter membrane should not be too strong, so that the CTCs could be kept on the top of membrane. Taken together, the GFP-based assays could detect the CTCs clusters, which are meaningful in reflecting the ability of metastasis, while this important information cannot be achieved by bioluminescence assay.

Thirdly, the increased speed of CTCs is not as fast as that of original source of lung metastases. The result showed that while at the 7 day intervals, the CTCs source site of lung GFP-Luc-4T1 metastases increased at a 10 time-log speed as measured in vivo with bioluminescence imaging (Fig. 6D), the CTCs increased only by a few (Fig. 6E). We are puzzled by this result that the CTCs increasing speed is not proportional to the fast growing lung metastases. Some studies showed that the number of CTCs in bloodstream had a peak followed by a decrease $(24,28-31)$; other researchers observed the number of CTCs in bloodstream had two peaks $(28,29)$, but that was not observed in this study. Sasportas et al consider that dynamic change of CTCs is not correlated with the growth of primary tumor (24), which is consistent with our observation. We speculate that the mechanism underlying this phenomenon may be: in early stage, a small amount of tumor cells have released into bloodstream to be CTCs, and the number of tumor cells in bloodstream gradually increased along with the growth of the original tumor. The immune system could be activated by the increasing CTCs, which in turn could kill CTCs and suppress their increasing.
However, while the newly generated immune cells could effectively kill a single CTC or cluster, they are not sufficient to kill the solid tumor or metastases due to the special tumor microenvironment that blocks the immune cells to penetrate or kill solid tumor cells.

Taken together, this study compared the sensitivity of three CTC detection methods side-by-side. Due to each of them having advantages and disadvantages, researchers should select the assays based on the needs of the study. For example, if the study is focused on the effect of certain agent on the suppression of CTCs, then the bioluminescence assay could be used; if the study is focused on dissemination mechanism of CTCs, then the GFP-based assays could be selected and used according to the laboratory condition and researcher's technical experience. Combination of both bioluminescence assay and GFP-based assay would certainly generate more information for the dissemination of cancer cells in terms of their quantity and malignant quality.

Further investigation of the balance between the CTCs and immune response might reveal the mechanism of complicated metastasis process and generate new strategy to control metastasis.

\section{Acknowledgements}

This study was supported in part by grant support from the China Science and Technology Fund (2015 DFA31770) to J.L.; Fujian Medical University Affiliated 1st Hospital fund to L.Z. (2013GXLJRC); Fujian Education Ministry (2013-58), Fujian Association for international exchange of personnel funds (W13350000137) to L.Z. and the Key Lab Fund from Fujian Medical University (0000-081919) to Fujian Key Laboratory of Individualized Active Immunotherapy and to Key Laboratory of Radiation Biology of Fujian Province University. We thank Kate Casey-Sawicki and Shimin Zhang for editing and preparing this manuscript for publication.

\section{References}

1. Ferlay J, Shin HR, Bray F, Forman D, Mathers C and Parkin DM: Estimates of worldwide burden of cancer in 2008: GLOBOCAN 2008. Int J Cancer 127: 2893-2917, 2010.

2. Sasportas LS and Gambhir SS: Imaging circulating tumor cells in freely moving awake small animals using a miniaturized intravital microscope. PLoS One 9: e86759, 2014.

3. Hayashi N, Nakamura S, Tokuda Y, Shimoda Y, Yagata H, Yoshida A, Ota H, Hortobagyi GN, Cristofanilli $M$ and Ueno NT: Prognostic value of HER2-positive circulating tumor cells in patients with metastatic breast cancer. Int J Clin Oncol 17: 96-104, 2012.

4. Lucci A, Hall CS, Lodhi AK, Bhattacharyya A, Anderson AE, Xiao L, Bedrosian I, Kuerer HM and Krishnamurthy S: Circulating tumour cells in non-metastatic breast cancer: A prospective study. Lancet Oncol 13: 688-695, 2012.

5. Rack B, Schindlbeck C, Jückstock J, Andergassen U, Hepp P, Zwingers T, Friedl TW, Lorenz R, Tesch H, Fasching PA, et al; SUCCESS Study Group: Circulating tumor cells predict survival in early average-to-high risk breast cancer patients. J Natl Cancer Inst 106: 106, 2014.

6. Bidard FC, Peeters DJ, Fehm T, Nolé F, Gisbert-Criado R, Mavroudis D, Grisanti S, Generali D, Garcia-Saenz JA, Stebbing J, et al: Clinical validity of circulating tumour cells in patients with metastatic breast cancer: A pooled analysis of individual patient data. Lancet Oncol 15: 406-414, 2014.

7. Scott J, Kuhn P and Anderson AR: Unifying metastasis - integrating intravasation, circulation and end-organ colonization. Nat Rev Cancer 12: 445-446, 2012. 
8. Marrinucci D, Bethel K, Bruce RH, Curry DN, Hsieh B, Humphrey M, Krivacic RT, Kroener J, Kroener L, Ladanyi A, et al: Case study of the morphologic variation of circulating tumor cells. Hum Pathol 38: 514-519, 2007.

9. Jiao LR, Apostolopoulos C, Jacob J, Szydlo R, Johnson N, Tsim N, Habib NA, Coombes RC and Stebbing J: Unique localization of circulating tumor cells in patients with hepatic metastases. J Clin Oncol 27: 6160-6165, 2009

10. Valastyan S and Weinberg RA: Tumor metastasis: Molecular insights and evolving paradigms. Cell 147: 275-292, 2011.

11. Alix-Panabières $C$ and Pantel K: Circulating tumor cells: Liquid biopsy of cancer. Clin Chem 59: 110-118, 2013.

12. Kraan J, Sleijfer S, Strijbos MH, Ignatiadis M, Peeters D, Pierga JY, Farace F, Riethdorf S, Fehm T, Zorzino L, et al: External quality assurance of circulating tumor cell enumeration using the CellSearch( $\left({ }^{\circ}\right)$ system: A feasibility study. Cytometry B Clin Cytom 80: 112-118, 2011.

13. Mostert B, Kraan J, Bolt-de Vries J, van der Spoel P, Sieuwerts AM, Schutte M, Timmermans AM, Foekens R, Martens JW, Gratama JW, et al: Detection of circulating tumor cells in breast cancer may improve through enrichment with antiCD146. Breast Cancer Res Treat 127: 33-41, 2011.

14. Pantel K, Alix-Panabières $C$ and Riethdorf $S$ : Cancer micrometastases. Nat Rev Clin Oncol 6: 339-351, 2009.

15. Stott SL, Hsu CH, Tsukrov DI, Yu M, Miyamoto DT, Waltman BA, Rothenberg SM, Shah AM, Smas ME, Korir GK, et al: Isolation of circulating tumor cells using a microvortexgenerating herringbone-chip. Proc Natl Acad Sci USA 107: 18392-18397, 2010.

16. Pinzani P, Salvadori B, Simi L, Bianchi S, Distante V, Cataliotti L, Pazzagli $\mathrm{M}$ and Orlando $\mathrm{C}$ : Isolation by size of epithelial tumor cells in peripheral blood of patients with breast cancer: Correlation with real-time reverse transcriptase-polymerase chain reaction results and feasibility of molecular analysis by laser microdissection. Hum Pathol 37: 711-718, 2006.

17. Wong NS, Kahn HJ, Zhang L, Oldfield S, Yang LY, Marks A and Trudeau ME: Prognostic significance of circulating tumour cells enumerated after filtration enrichment in early and metastatic breast cancer patients. Breast Cancer Res Treat 99: 63-69, 2006.

18. Zieglschmid V, Hollmann C and Böcher O: Detection of disseminated tumor cells in peripheral blood. Crit Rev Clin Lab Sci 42: 155-196, 2005

19. Basiji DA, Ortyn WE, Liang L, Venkatachalam V and Morrissey P: Cellular image analysis and imaging by flow cytometry. Clin Lab Med 27: 653-670, viii, 2007.

20. Van der Auwera I, Peeters D, Benoy IH, Elst HJ, Van Laere SJ, Prové A, Maes H, Huget P, van Dam P, Vermeulen PB, et al: Circulating tumour cell detection: A direct comparison between the CellSearch System, the AdnaTest and CK-19/mammaglobin RT-PCR in patients with metastatic breast cancer. Br J Cancer 102: $276-284,2010$
21. Krebs MG, Hou JM, Sloane R, Lancashire L, Priest L, Nonaka D, Ward TH, Backen A, Clack G, Hughes A, et al: Analysis of circulating tumor cells in patients with non-small cell lung cancer using epithelial marker-dependent and -independent approaches. J Thorac Oncol 7: 306-315, 2012.

22. Gervasoni A, Sandri MT, Nascimbeni R, Zorzino L, Cassatella MC, Baglioni L, Panigara S, Gervasi M, Di Lorenzo D and Parolini O: Comparison of three distinct methods for the detection of circulating tumor cells in colorectal cancer patients. Oncol Rep 25: 1669-1703, 2011.

23. Rashid OM, Nagahashi M, Ramachandran S, Dumur CI, Schaum JC, Yamada A, Aoyagi T, Milstien S, Spiegel S and Takabe K: Is tail vein injection a relevant breast cancer lung metastasis model? J Thorac Dis 5: 385-392, 2013.

24. Sasportas LS, Hori SS, Pratx G and Gambhir SS: Detection and quantitation of circulating tumor cell dynamics by bioluminescence imaging in an orthotopic mammary carcinoma model. PLoS One 9: e105079, 2014

25. James ML and Gambhir SS: A molecular imaging primer: Modalities, imaging agents, and applications. Physiol Rev 92: 897-965, 2012.

26. Choi JW, Kim JK, Yang YJ, Kim P, Yoon KH and Yun SH: Urokinase exerts antimetastatic effects by dissociating clusters of circulating tumor cells. Cancer Res 75: 4474-4482, 2015.

27. Aceto N, Bardia A, Miyamoto DT, Donaldson MC, Wittner BS, Spencer JA, Yu M, Pely A, Engstrom A, Zhu H, et al: Circulating tumor cell clusters are oligoclonal precursors of breast cancer metastasis. Cell 158: 1110-1122, 2014.

28. Juratli MA, Sarimollaoglu M, Nedosekin DA, Melerzanov AV, Zharov VP and Galanzha EI: Dynamic fluctuation of circulating tumor cells during cancer progression. Cancers (Basel) 6: 128-142, 2014.

29. Bonnomet A, Syne L, Brysse A, Feyereisen E, Thompson EW, Noël A, Foidart JM, Birembaut P, Polette M and Gilles C: A dynamic in vivo model of epithelial-to-mesenchymal transitions in circulating tumor cells and metastases of breast cancer. Oncogene 31: 3741-3753, 2012.

30. Schmidt CM, Settle SL, Keene JL, Westlin WF, Nickols GA and Griggs DW: Characterization of spontaneous metastasis in an aggressive breast carcinoma model using flow cytometry. Clin Exp Metastasis 17: 537-544, 1999.

31. Aslakson CJ and Miller FR: Selective events in the metastatic process defined by analysis of the sequential dissemination of subpopulations of a mouse mammary tumor. Cancer Res 52: 1399-1405, 1992 